\title{
Proposta de instrumento para avaliação da governança organizacional em uma instituição do setor público
}

\section{Nildete dos Passos Oliveira}

Universidade de Brasília (UnB), Brasília, DF - Brasil

\section{Pedro Carlos Resende Junior}

Universidade de Brasília (UnB), Brasília, DF-Brasil

Este estudo tem como objetivo propor um instrumento de avaliação da governança organizacional para uma instituição pública e foi construído a partir de uma abordagem qualitativa e quantitativa. Foi utilizada a perspectiva organizacional da governança, a qual está relacionada a incentivos, decisões administrativas e funcionamento da organização. A coleta de dados ocorreu por meio de análise documental, entrevistas semiestruturadas com especialistas e gestores de uma instituição de estudo e questionários. Para análise dos dados, foram empregadas as técnicas de análise documental e análise de conteúdo categorial, com avaliação da saturação teórica e do coeficiente de validação de conteúdo. Mediante análise dos referenciais de governança e da revisão de literatura foram definidas três categorias descritivas do constructo: gestão estratégica, pessoas e accountability, agrupando variáveis de governança. Após análise das entrevistas, observou-se a predominância da categoria gestão estratégica, seguida por pessoas e accountability. Então, o instrumento de avaliação, construído com 16 variáveis e contendo 58 itens, foi avaliado por sete juízes, resultando na proposta de instrumento de avaliação da governança organizacional.

Palavras-chave: governança, setor público, avaliação 


\section{Propuesta de instrumento para evaluación la gobernanza organizacional en una institución del sector público}

Este estudio tiene como objetivo proponer un instrumento de evaluación de la gobernanza organizacional para una institución publica y fue construido a partir de un enfoque cualitativo y cuantitativo. Se utilizó la perspectiva organizacional de la gobernanza, la cual está relacionada a incentivos, decisiones administrativas y funcionamiento de la organización. La recolección de datos ocurrió por medio de análisis documental, entrevistas semiestructuradas con especialistas y gestores de una institución de estudio y cuestionarios. Para el análisis de los datos, se emplearon las técnicas de análisis documental y análisis de contenido categorial, con evaluación de la saturación teórica y del coeficiente de validación de contenido. En el análisis de los referentes de gobernanza y de la revisión de literatura se definieron tres categorías descriptivas del constructo: gestión estratégica, personas y accountability, agrupando variables de gobernanza. Después del análisis de las entrevistas, se observó el predominio de la categoría gestión estratégica, seguida por personas y accountability. Entonces, el instrumento de evaluación, construido con 16 variables y conteniendo 58 ítems, fue evaluado por siete jueces, resultando en la propuesta de instrumento de evaluación de la gobernanza organizacional.

Palabras clave: gobernanza, sector público, evaluación

\section{Proposal of an instrument for assessment of organizational governance in a public sector institution}

This study aims to propose an instrument of assessment of organizational governance for a public institution and was built from a qualitative and quantitative approach. An organizational approach to governance was used, which is related to incentives, administrative decisions and the functioning of the organization. Data collection took place through documental analysis, semi-structured interviews with specialists and managers of the institution and questionnaires. For data analysis, the techniques of document analysis and categorical content analysis, with assessment of theoretical saturation and calculation of content validation were used. Through analysis of the governance frameworks and the literature review, three descriptive categories of the construct were defined: strategic management, people and accountability, grouping governance variables. After analyzing the interviews, it was observed the predominance of the category strategic management, followed by people and accountability. Then, the evaluation instrument, constructed with 16 variables and containing 58 items, was assessment by seven judges, resulting in the proposal of an organizational governance assessment tool.

Keywords: governance, public sector, assessment 


\section{Introdução}

O termo governança foi inicialmente aplicado no contexto de empresas privadas (CAVAlCANTE; De LuCA, 2013) para designar "o conjunto de práticas e estudos que tratam da forma como a empresa é administrada e a relação com os diversos indivíduos em sua atuação" (SoUZA, 2013, p. 60). O referido termo também foi adotado no setor público, embora alguns autores adotem a expressão governança organizacional, como será tratada no decorrer desse estudo, para diferenciá-lo do termo aplicado ao setor privado (FONTESFILHO, 2003; TRIBUNAL DE CONTAS DA UNIÃO, 2014)

A governança aplicada ao setor público também envolve a noção de como as decisões políticas e administrativas são tomadas pelos gestores, quanto ao funcionamento de sistemas governamentais, a influência de instituições formais e informais nas instituições e como o Estado se relaciona com a sociedade (GRINDLE, 2017).

Além disso, a governança também pode ser entendida como direcionadora de resultado, uma vez que está orientada para o desempenho e a geração de valor público, sendo tais conceitos entendidos como os esforços empregados para o alcance dos resultados e aqueles auferidos pelos beneficiários das ações do poder público (MARTINS; MARINI, 2014). O valor público acaba por direcionar a ação da governança para o objeto da organização ("o que" deve ser gerado), para o objetivo ("para quê") e para o sujeito ("para quem") das ações governamentais (MARTINS; MARINI, 2014, p. 130).

Nesse sentido, identificar e compreender o funcionamento das estruturas e dos mecanismos de governança de uma organização pode contribuir para aprimorar o desempenho da organização e permitir um melhor direcionamento dos recursos, visando à geração de valor público (MARTINS; MARINI, 2014; OlIVEIRA; PISA, 2015). Para isso, e utilizando como estudo de caso uma entidade autárquica vinculada ao Ministério da Educação, este estudo se propõe a apresentar um instrumento de avaliação de governança organizacional a partir do entendimento do objeto de estudo, da realização de entrevistas e da validação do instrumento por especialistas e gestores da organização utilizada como estudo de caso. 
Cabe ressaltar que a maioria dos estudos relacionados à governança organizacional, identificados por meio de revisão de literatura, direciona-se a análises e propostas ao contexto do setor privado (BENEDicto et al., 2013; Flórez-PARRA; LóPEZ-PÉrez; LóPEZHernández, 2014; Hora; Oliveira; Forte, 2014; Mazzioni et al., 2015; Ribeiro, 2014; RIBEIRo et al., 2012; RIBEIRO; SANTOS, 2015; SCHIEHLL; MARTINS, 2016) e não apresenta proposta de instrumento de avaliação da governança em organizações, razão pela qual este estudo se apresenta como instrumento alternativo para preencher a lacuna teórica.

A escolha da instituição como estudo de caso se justifica pelo protagonismo da organização no contexto de avaliação da educação brasileira, sendo responsável por elaborar e disseminar estudos, pesquisas, levantamentos estatísticos, avaliações e exames da educação básica e superior do país, além de subsidiar a formulação, a implementação e o monitoramento de políticas públicas educacionais (BRASIL, 1997) e atualmente é responsável por aferir a evolução das 20 metas do Plano Nacional de Educação (PNE) do período 2014-2024. Dado o relevante interesse público na instituição e considerando que a governança permite agregar valor à organização (MARQUES, 2007), vislumbra-se que a avaliação da estrutura e dos mecanismos de governança pode aprimorar a avaliação realizada sobre a educação brasileira e melhorar a qualidade das atividades da organização, traduzindo em benefícios para a sociedade.

\section{Referencial teórico}

A governança aplicada ao setor público pode ser operacionalizada em três níveis: macro, meso e micro - sendo que para cada nível faz-se necessário um modelo ajustado de governança (SPANHOVE; VERHOEST, 2007). O nível macro está relacionado ao ambiente macro estatal, centrado na governança de todo o setor público e nas relações entre o Congresso, o Poder Executivo e o aparelho administrativo. No nível meso encontra-se o setor político, onde departamentos e agências, lideradas por um ministro, focam na execução de políticas públicas. O nível micro refere-se ao nível da organização, chefiada por um gestor e, em alguns casos, por um conselho de administração, cujas ações devem estar vinculadas aos objetivos de governo do nível macro, sob pena de, no limite, serem ineficazes (SPANHOVE; VERHOEST, 2007). 
A governança no nível micro ou governança organizacional, portanto, está relacionada a competências, estruturas e instrumentos para orientar, controlar, monitorar e explicar o comportamento de indivíduos dentro de um departamento ou agência, de forma a alcançar os objetivos organizacionais de forma eficiente, efetiva e transparente (SPANHOVE; VERHOEST, 2007).

Para compreender o movimento de governança nas organizações, a partir de mudanças ocorridas em suas estruturas, nos mecanismos de funcionamento e da interação com outros atores, foram utilizadas neste estudo a Teoria Institucional e a Teoria dos Stakeholders.

A Teoria Institucional permite compreender o processo de legitimação das práticas de governança e das mudanças organizacionais que ocorrem na incorporação de tais práticas pelas organizações, especialmente quanto ao processo isomórfico, com vistas à melhoria do desempenho. De acordo com DiMaggio e Powell (1983), há três mecanismos de mudança isomórfica: coercitivo, mimético e o normativo.

Pelo mecanismo coercitivo, a mudança em uma organização deriva de influências políticas e pressões formais e informais exercidas por outras organizações com domínio de recursos escassos, especialmente recursos financeiros. O isomorfismo mimético resulta da adoção de práticas que foram reconhecidamente bem-sucedidas ou legitimadas por outras organizacionais, ocorrendo quando há incerteza de se adotar uma prática inovativa na organização. O terceiro mecanismo, o normativo, está associado à mudança decorrente da influência de forças profissionais - membro ou classe de determinada profissão - que institui um conjunto de normas e procedimentos a serem obrigatoriamente adotados pela organização.

Se, por um lado, as mudanças organizacionais são impulsionadas por mecanismos institucionais, por outro, os indivíduos possuem um papel importante, porque é a partir dos significados atribuídos por eles à sua realidade que se determinam e se orientam as ações organizacionais (CARVAlHo; VIEIRA; SILVA, 2012). A influência de indivíduos e outras organizações sob uma instituição também pode ser observada à luz da Teoria dos Stakeholders, ou Teoria das Partes Interessadas, na qual a expressão stakeholders ou partes interessadas pode ser entendida como grupos ou indivíduos que afetam e são afetados pelo alcance dos objetivos da organização (FREEMAN; REED, 1983). 
Em relação à gestão dos stakeholders, Gomes (2005) aponta que as principais questões nesse contexto estão relacionadas à identificação das principais partes interessadas, à identificação do tipo de influência ou interesse que conecta as principais partes interessadas à organização e à identificação do tipo de estratégia que a organização pode utilizar para gerenciar as relações com as partes interessadas.

O modelo de Mitchell e Wood (1997), de classificação dos stakeholders e escolha de estratégia de gestão, considera três atributos principais de classificação - poder, legitimidade e urgência - que, quando combinados, originam diferentes tipos de partes interessadas e que demandam uma reação diferente por parte da organização. A reação vai depender da quantidade de atributos que cada stakeholder possuir, seja inicialmente ou quando incorporados pela parte interessada durante o processo de interação com a organização. Quanto mais atributos o stakeholder apresentar, maior o potencial de influenciar as decisões das organizações e diferente tratamento deve ser dispensado a ele.

Retornando à governança, para a identificação das categorias de análise de um modelo de governança aplicado à organização pública, considerou-se o entendimento do Tribunal de Contas da União (TCU, 2014) de que um sistema de governança no setor público envolve estruturas administrativas (instâncias), processos de trabalho, instrumentos (ferramentas, documentos etc.), fluxo de informações e comportamento de pessoas envolvidas, direta ou indiretamente, na avaliação, no direcionamento e no monitoramento da organização, aqui incluídos os stakeholders. Impactando o sistema de governança, há eventos potenciais que podem comprometer o alcance dos objetivos organizacionais e que devem ser identificados, tratados, e monitoramentos por meio de adequada gestão de riscos (IFAC, 2001). Nesse sentido, a primeira categoria do modelo, denominada gestão estratégica, é adequada para abordar elementos relacionados à lógica de funcionamento da organização.

Uma segunda categoria relevante para a governança refere-se aos agentes que atuam na organização e foi intitulada como pessoas. A atuação das pessoas na organização é fundamental para a governança (MARQUES, 2007; TCU, 2014) e seu comportamento pode afetar, positiva ou negativamente, os resultados das organizações (MINISTÉRIO DO Planejamento, 2014). Sobre esse aspecto, a Instrução Normativa no 01 (CGU/MPOG, 2016) 
define como princípio de governança aplicável a órgãos e entidades do Poder Executivo federal o compromisso dos agentes em atuar segundo os valores éticos.

A gestão de pessoas, de forma abrangente, envolve selecionar, capacitar e avaliar o desempenho dos agentes, em especial a alta administração (IFAC, 2001; TCU, 2014), com vistas ao melhor desempenho da organização. Além disso, o adequado envolvimento da liderança é crucial para a boa governança e a prestação de serviços (NGOEPE; NGULUBE, 2013), uma vez que entre suas responsabilidades estão a implementação, a avaliação e a melhoria das estratégias da organização (SPANHOVE; VERHOEST, 2007). Ademais, a ética do comportamento desses agentes influencia a atuação dos demais agentes da organização (CHERMAN; TOMEI, 2005).

Outra categoria relevante para a governança está relacionada aos mecanismos de accountability da organização e envolve a transparência, a responsabilização dos agentes por meios de ações de controle e a prestação de contas (KOPPELL, 2005; PINHO; SACRAMENTO, 2009; ROCHA, 2009). Sobre a palavra accountability, Pinho e Sacramento (2009) se propuseram a identificar a tradução para a língua pátria, mas constataram a impossibilidade de uma única tradução, tendo em vista que o conceito ainda está em construção no país. Não obstante, os autores apontam que o conceito envolve: responsabilidade (objetiva e subjetiva), controle, transparência, obrigação de prestação de contas, justificativas para as ações que foram ou deixaram de ser empreendidas, premiação e/ou castigo (PINHO; SACRAMENTO, 2009).

As categorias, variáveis e elementos relacionados que formam a caracterização do modelo de governança organizacional, a partir da revisão de literatura, podem ser observados no Quadro a seguir. 


\section{Quadro 1 | Categorias e variáveis do modelo de governança}

\begin{tabular}{|c|c|c|c|c|c|}
\hline \# & Categoria & $\begin{array}{l}\text { Descrição da } \\
\text { categoria }\end{array}$ & Variável & $\begin{array}{l}\text { Elemento relacionado à } \\
\text { variável }\end{array}$ & Referência \\
\hline \multirow{11}{*}{1} & \multirow{11}{*}{$\begin{array}{l}\text { Gestão } \\
\text { Estratégica }\end{array}$} & \multirow{11}{*}{$\begin{array}{l}\text { Envolve } \\
\text { aspectos } \\
\text { relacionados ao } \\
\text { funcionamento } \\
\text { da organização }\end{array}$} & \multirow{3}{*}{$\begin{array}{l}\text { Instâncias } \\
\text { administrativas de } \\
\text { apoio à governança }\end{array}$} & $\begin{array}{l}\text { Configuração } \\
\text { do Conselho de } \\
\text { Administração }\end{array}$ & $\begin{array}{l}\text { Blank e Van Hulst (2011); Chahal } \\
\text { e Kumari (2013); Jarboui, Forget } \\
\text { e Boujelbene (2014); Mswaka e } \\
\text { Olu (2015); Nkundabanyanga, } \\
\text { Tauringana e Muhwezi (2015) }\end{array}$ \\
\hline & & & & $\begin{array}{l}\text { Comitês de apoio à } \\
\text { governança }\end{array}$ & $\begin{array}{l}\text { IFAC (2001); Lei n. } 13.303 \text { (Brasil, } \\
\text { 2016); }\end{array}$ \\
\hline & & & & $\begin{array}{l}\text { Atuação de auditoria } \\
\text { interna }\end{array}$ & $\begin{array}{l}\text { Bello (2013); Chahal e Kumari } \\
\text { (2013); Ellwood e Garcia-Lacalle } \\
\text { (2015); IFAC (2001); Lei n. 13.303 } \\
\text { (Brasil, 2016); Schelker (2013); TCU } \\
\text { (2014) }\end{array}$ \\
\hline & & & $\begin{array}{l}\text { Processos } \\
\text { organizacionais }\end{array}$ & $\begin{array}{l}\text { Gerenciamento de } \\
\text { informações }\end{array}$ & $\begin{array}{l}\text { ANAO (2014); IFAC (2001); IFAC/ } \\
\text { CIPFA (2014); Ngoepe e Ngulube } \\
\text { (2013); Schelker (2013); Romolini, } \\
\text { Gori e Fissi (2015); OCDE (2015) }\end{array}$ \\
\hline & & & \multirow{2}{*}{$\begin{array}{l}\text { Estratégia da } \\
\text { organização }\end{array}$} & $\begin{array}{l}\text { Definição e } \\
\text { implementação de } \\
\text { Gestão Estratégica }\end{array}$ & $\begin{array}{l}\text { ANAO (2014); IFAC/CIPFA (2014); } \\
\text { Gnan et. al (2013); Kim e Kim } \\
\text { (2016), Loi (2016); Meintjes e } \\
\text { Grobler (2014); TCU (2014) }\end{array}$ \\
\hline & & & & Planejamento Estratégico & ANAO (2014) \\
\hline & & & Gestão de Riscos & $\begin{array}{l}\text { Implementação e } \\
\text { avaliação da Gestão de } \\
\text { Riscos }\end{array}$ & $\begin{array}{l}\text { ANAO (2014); IFAC/CIPFA (2014); } \\
\text { Lei n. } 13.303 \text { (Brasil, 2016); TCU } \\
\text { (2014) }\end{array}$ \\
\hline & & & \multirow[b]{2}{*}{ Stakeholders } & $\begin{array}{l}\text { Definição e divulgação } \\
\text { dos canais de } \\
\text { comunicação }\end{array}$ & $\begin{array}{l}\text { ANAO (2014); IFAC (2001); OCDE } \\
\text { (2015); TCU (2014) }\end{array}$ \\
\hline & & & & $\begin{array}{l}\text { Fomento à participação } \\
\text { das partes Interessadas }\end{array}$ & $\begin{array}{l}\text { ANAO (2014); IFAC (2001); Gnan } \\
\text { et. al (2013); IFAC/CIPFA (2014); } \\
\text { Kim e Kim (2016); Lei n. 13.303 } \\
\text { (BRASIL, 2016); Loi (2016); } \\
\text { Meintjes e Grobler (2014); OCDE } \\
\text { (2015); TCU (2014) }\end{array}$ \\
\hline & & & Stakeholders & $\begin{array}{l}\text { Atendimento dos } \\
\text { interesses das partes } \\
\text { interessadas }\end{array}$ & $\begin{array}{l}\text { ANAO (2014); IN n. } 01 \text { (CGU/ } \\
\text { MPOG, 2016); OCDE (2015); TCU } \\
\text { (2014) }\end{array}$ \\
\hline & & & Parcerias & Atuação compartilhada & $\begin{array}{l}\text { ANAO (2014); IFAC/CIPFA (2014); } \\
\text { TCU (2014) }\end{array}$ \\
\hline
\end{tabular}




\begin{tabular}{|c|c|c|c|c|c|}
\hline \# & Categoria & $\begin{array}{l}\text { Descrição da } \\
\text { categoria }\end{array}$ & Variável & $\begin{array}{l}\text { Elemento relacionado à } \\
\text { variável }\end{array}$ & Referência \\
\hline \multirow{10}{*}{2} & \multirow{10}{*}{ Pessoas } & \multirow{10}{*}{$\begin{array}{l}\text { Envolve } \\
\text { aspectos } \\
\text { relacionados } \\
\text { às pessoas } \\
\text { que atuam na } \\
\text { organização }\end{array}$} & & $\begin{array}{l}\text { Definição de diretrizes e } \\
\text { políticas da organização }\end{array}$ & $\begin{array}{l}\text { IFAC (2001); IFAC/CIPFA (2014); } \\
\text { TCU (2014) }\end{array}$ \\
\hline & & & Exercício da liderança & $\begin{array}{l}\text { Responsabilidade } \\
\text { pelo direcionamento, } \\
\text { monitoramento e } \\
\text { avaliação da organização }\end{array}$ & $\begin{array}{l}\text { IFAC (2001); IFAC/CIPFA (2014); IN } \\
\text { n. } 01 \text { (CGU/MPOG, 2016); OCDE } \\
\text { (2015); TCU (2014); }\end{array}$ \\
\hline & & & \multirow{3}{*}{ Gestão de Pessoas } & Seleção de pessoas & $\begin{array}{l}\text { IFAC (2001); Lei n. } 13.303 \text { (Brasil, } \\
\text { 2016); Schelker (2013); TCU (2014) }\end{array}$ \\
\hline & & & & Capacitação de pessoas & $\begin{array}{l}\text { ANAO (2014); IFAC (2001); IFAC/ } \\
\text { CIPFA (2014); IN n. } 01 \text { (CGU/ } \\
\text { MPOG, 2016); TCU (2014); }\end{array}$ \\
\hline & & & & Avaliação de pessoas & $\begin{array}{l}\text { Lei n. } 13.303 \text { (Brasil, 2016); TCU } \\
\text { (2014) }\end{array}$ \\
\hline & & & $\begin{array}{l}\text { Papéis e } \\
\text { responsabilidades }\end{array}$ & $\begin{array}{l}\text { Definição de atribuições } \\
\text { e de responsabilidades }\end{array}$ & $\begin{array}{l}\text { ANAO (2014); IFAC (2001); OCDE } \\
\text { (2015); Oberoi (2013); TCU (2014) }\end{array}$ \\
\hline & & & $\begin{array}{l}\text { Remuneração da alta } \\
\text { administração }\end{array}$ & $\begin{array}{l}\text { Definição da } \\
\text { remuneração da alta } \\
\text { administração }\end{array}$ & IFAC (2001); TCU (2014) \\
\hline & & & \multirow{3}{*}{$\begin{array}{l}\text { Padrão } \\
\text { comportamental ético }\end{array}$} & Atuação ética & $\begin{array}{l}\text { IFAC/CIPFA (2014); IN n. } 01 \text { (CGU/ } \\
\text { MPOG, 2016); Jarboui, Forget e } \\
\text { Boujelbene (2014); Subramaniam, } \\
\text { Stewart e Shulman (2013) }\end{array}$ \\
\hline & & & & $\begin{array}{l}\text { Adoção de Código de } \\
\text { Ética }\end{array}$ & $\begin{array}{l}\text { IFAC (2001); IFAC/CIPFA (2014); } \\
\text { Lei n. 13.303 (Brasil, 2016); OCDE } \\
\text { (2015); TCU (2014) }\end{array}$ \\
\hline & & & & $\begin{array}{l}\text { Julgamento imparcial na } \\
\text { tomada de decisão }\end{array}$ & IFAC (2001); OCDE (2015) \\
\hline
\end{tabular}




\begin{tabular}{|c|c|c|c|c|c|}
\hline \# & Categoria & $\begin{array}{l}\text { Descrição da } \\
\text { categoria }\end{array}$ & Variável & $\begin{array}{l}\text { Elemento relacionado à } \\
\text { variável }\end{array}$ & Referência \\
\hline \multirow{9}{*}{3} & \multirow{9}{*}{ Accountability } & \multirow{9}{*}{$\begin{array}{l}\text { Envolve } \\
\text { transparência, } \\
\text { responsabiliza- } \\
\text { ção e prestação } \\
\text { de contas da } \\
\text { organização }\end{array}$} & \multirow{3}{*}{ Transparência } & $\begin{array}{l}\text { Transparência de dados e } \\
\text { informações }\end{array}$ & $\begin{array}{l}\text { ANAO (2014); IFAC (2001); IFAC/ } \\
\text { CIPFA (2014) IN n. } 01 \text { (CGU/MPOG, } \\
\text { 2016); Lei n. 13.303 (Brasil, 2016); } \\
\text { OCDE (2015); TCU (2014) }\end{array}$ \\
\hline & & & & Avaliação de satisfação & ANAO (2014); TCU (2014) \\
\hline & & & & $\begin{array}{l}\text { Monitoramento } \\
\text { e avaliação de } \\
\text { desempenho da } \\
\text { organização }\end{array}$ & $\begin{array}{l}\text { ANAO (2014); IFAC (2001); IFAC/ } \\
\text { CIPFA (2014); TCU (2014) }\end{array}$ \\
\hline & & & \multirow{4}{*}{ Responsabilização } & $\begin{array}{l}\text { Conformidade com leis e } \\
\text { regulamentos }\end{array}$ & IFAC (2001) \\
\hline & & & & $\begin{array}{l}\text { Conformidade } \\
\text { orçamentária e financeira }\end{array}$ & IFAC (2001); IFAC/CIPFA (2014) \\
\hline & & & & $\begin{array}{l}\text { Gerenciamento do } \\
\text { conflito de interesse }\end{array}$ & $\begin{array}{l}\text { ANAO (2014); IFAC (2001), OCDE } \\
\text { (2015); TCU (2014) }\end{array}$ \\
\hline & & & & $\begin{array}{l}\text { Apuração de } \\
\text { irregularidades }\end{array}$ & IFAC (2001); TCU (2014) \\
\hline & & & \multirow[t]{2}{*}{ Prestação de contas } & $\begin{array}{l}\text { Prestação de contas } \\
\text { periódicas }\end{array}$ & $\begin{array}{l}\text { ANAO (2014); IFAC (2001); IFAC/ } \\
\text { CIPFA (2014); IN n. } 01 \text { (CGU/ } \\
\text { MPOG, 2016); Ngoepe e Ngulube } \\
\text { (2013); Romolini, Gori e Fissi } \\
\text { (2015); Schelker (2013); OCDE } \\
\text { (2015); TCU (2014) }\end{array}$ \\
\hline & & & & $\begin{array}{l}\text { Realização de auditoria } \\
\text { anual }\end{array}$ & OCDE (2015) \\
\hline
\end{tabular}

Fonte: dados da pesquisa.

Cabe ressaltar que a revisão de literatura foi realizada a partir de pesquisas em periódicos nacionais e internacionais e nos principais referenciais de governança aplicados ao setor público. O quadro orientou o roteiro da coleta de dados primários, conforme especificado no tópico seguinte. 


\section{Método}

Trata-se de estudo qualitativo e quantitativo para a proposição de um instrumento de avaliação de governança organizacional por meio de procedimentos metodológicos de investigação documental, revisão de literatura, realização de entrevistas com especialistas sobre o tema e com gestores de uma organização pública, e validação do instrumento de avaliação. Optou-se como estratégia de pesquisa o estudo de caso em uma instituição pública federal, constituída como autarquia e vinculada ao Ministério da Educação, uma vez que se busca examinar um fenômeno contemporâneo dentro do seu contexto por meio do emprego de diversos meios de obtenção de dados, como entrevistas, análise documental e observações (YıN, 2001).

$\mathrm{Na}$ primeira etapa da pesquisa, de natureza qualitativa, e a fim de identificar categorias e variáveis de governança organizacional no setor público, promoveu-se o levantamento da literatura e dos principais referenciais de governança aplicados ao setor público. Em seguida, a partir das descrições das categorias e das variáveis apresentadas no Quadro 1, foi definido um roteiro de entrevistas e aplicado em entrevistas semiestruturadas com 14 especialistas sobre o tema governança, sendo quatro gestores, três acadêmicos e sete auditores escolhidos por amostragem não probabilística. As entrevistas foram realizadas individualmente, de forma presencial, entre maio e setembro de 2017, tiveram duração média de uma hora e trinta minutos, foram gravadas, transcritas e analisadas por meio da técnica de análise de conteúdo categorial temática (BARDIN, 2011). A quantidade de entrevistas foi definida em função do ponto de saturação teórica (THIRY-CHERQUES, 2009), que permite a interrupção da realização de entrevista quando, após identificada redundância dos dados coletados, realiza-se, no mínimo, duas entrevistas adicionais para confirmar o ponto de saturação (FALQUETTO; FARIAS, 2016).

Além das 14 entrevistas realizadas com especialistas em governança, foram realizadas entrevistas também com gestores da organização de estudo com o objetivo de verificar categorias e variáveis do modelo de governança na realidade organizacional. Dessa forma, com autorização do mais alto dirigente da organização, o mesmo roteiro de entrevistas aplicado aos especialistas foi utilizado para realizar as entrevistas com oito gestores, sendo um representante de cada unidade do mais alto nível regimental da 
organização, entre agosto e outubro de 2017. No total, portanto, somando especialistas e gestores, foram realizadas 22 entrevistas.

Na análise das entrevistas, buscou-se verificar a presença das categorias e variáveis identificadas na etapa inicial do estudo, assim como novas categorias ou variáveis a partir dos relatos de cada entrevistado. Ao final, um instrumento de avaliação da governança organizacional foi construído e submetido à validação teórica e semântica de sete juízes, todos especialistas acadêmicos, para avaliação quanto aos critérios de clareza da linguagem, de pertinência prática e de relevância teórica dos itens elaborados, seguindo o método de validação pelo Coeficiente de Validade de Conteúdo (CVC), elaborado por Hernández Nieto (2002), onde:

(i) calcula-se a média $(\mathrm{Mx})$ das notas atribuídas pelos juízes em cada critério e para cada item, conforme apresentado abaixo, onde $\mathrm{i}=1$ representa a soma das notas dos juízes e j o número de juízes:

$$
\mathrm{Mx}=\frac{\sum_{\mathrm{i}=1}^{7} \mathrm{Xij}}{j}
$$

(ii) com base na média obtida anteriormente, apura-se o coeficiente de cada critério e em cada item ( $C V C i)$, onde $V \max =5$, uma vez que foi utilizada a escala Likert de 5 pontos:

$$
\mathrm{CVCi}=\frac{\mathrm{Mx}}{\mathrm{Vmax}}
$$

(iii) em seguida, deduz-se o erro (Pei) de o juiz atribuir notas aleatórias gerando uma concordância tendenciosa:

$$
\text { Pei }=\left(\frac{1}{J}\right)^{j}
$$

(iv) na sequência, obtém-se o coeficiente de cada item (CVCc):

$$
\text { CVCc }=\text { CVCi }- \text { Pei }
$$


(v) e finalmente, calcula-se o coeficiente total (CVCt) do instrumento de avaliação, para cada um dos três critérios, a partir da média dos coeficientes de validade de conteúdo dos itens do instrumento (Mccvi) descontado da média dos erros dos itens do instrumento:

$$
\text { CVCt }=\text { Mcvci }- \text { Mpei }
$$

Cada juiz foi instruído a atribuir nota de 1 a 5, em que os extremos, 1 e 5, expressavam a menor e a maior intensidade, respectivamente, de concordância do item avaliado em cada um dos três critérios. Adicionalmente, para cada item havia um campo de observações para aporte de comentário dos juízes.

Para validação e de acordo com Hernández Nieto (2002), considerando uma escala entre 0,0 e 1,00 de avaliação do CVCc e CVCt, itens com valores menores de 0,80 representam validade e concordância inaceitáveis; valores iguais ou maiores de 0,80 e menores de 0,90, são considerados com validade e concordância satisfatórias; e valores iguais ou acima de 0,90 até o limite de 1,00, apresentam validade e concordância excelentes (HERNÁNDEZ NiETO, 2002).

\section{Resultados}

A identificação de categorias e variáveis obtidas com o levantamento dos referenciais de governança e revisão de literatura foram apresentados no Quadro 1. $\mathrm{Na}$ etapa de entrevistas com especialistas, observou-se a ocorrência do ponto de saturação teórica em momentos distintos para cada categoria, como demonstrado na Tabela 1, e apesar da recomendação de apenas duas entrevistas adicionais para confirmação da saturação, optou-se por manter o cronograma de entrevistas agendado resultando em quatro entrevistas adicionais para a categoria Gestão Estratégica. 
Tabela 1 | Saturação das categorias

\begin{tabular}{lcccccccccccccc} 
Categorias & E1 & E2 & E3 & E4 & E5 & E6 & E7 & E8 & E9 & E10 & E11 & E12 & E13 & E14 \\
$\begin{array}{l}\text { Gestão } \\
\text { Estratégica }\end{array}$ & 1 & 1 & 1 & 1 & 1 & 1 & 1 & 1 & 0 & 1 & 0 & 0 & 0 & 0 \\
Pessoas & 1 & 1 & 1 & 1 & 0 & 1 & 1 & 0 & 1 & 0 & 0 & 0 & 0 & 0 \\
\hline Accountability & 1 & 1 & 0 & 1 & 0 & 0 & 0 & 0 & 0 & 0 & 0 & 0 & 0 & 0 \\
\hline $\begin{array}{l}\text { Fonte: dados da pesquisa. } \\
\end{array}$ & & & & & & & & & & & &
\end{tabular}

Fonte: dados da pesquisa.

Em relação à frequência com que categorias e variáveis foram mencionadas nos discursos dos entrevistados, obtiveram-se os dados da Tabela abaixo.

Tabela 2 | Frequência de entrevistas por categorias e variáveis

\begin{tabular}{|c|c|c|c|}
\hline Categorias & Variáveis & $\begin{array}{c}\text { Quantidade de } \\
\text { entrevistas por } \\
\text { variável }\end{array}$ & Percentual \\
\hline $\begin{array}{l}\text { Gestão } \\
\text { Estratégica }\end{array}$ & $\begin{array}{l}\text { Instâncias administrativas de apoio à } \\
\text { governança } \\
\text { Processos organizacionais } \\
\text { Apoio tecnológico } \\
\text { Práticas de governança } \\
\text { Estratégia da Organização } \\
\text { Gestão de riscos } \\
\text { Stakeholders } \\
\text { Parcerias }\end{array}$ & $\begin{array}{l}8 \\
18 \\
6 \\
3 \\
19 \\
21 \\
22 \\
21\end{array}$ & $\begin{array}{l}36 \% \\
82 \% \\
27 \% \\
14 \% \\
86 \% \\
95 \% \\
100 \% \\
95 \%\end{array}$ \\
\hline Pessoas & $\begin{array}{l}\text { Exercício da liderança } \\
\text { Gestão de pessoas } \\
\text { Papéis e responsabilidades } \\
\text { Remuneração da alta administração } \\
\text { Padrão comportamental ético }\end{array}$ & $\begin{array}{c}20 \\
22 \\
10 \\
0 \\
8\end{array}$ & $\begin{array}{c}91 \% \\
100 \% \\
45 \% \\
0 \% \\
36 \%\end{array}$ \\
\hline Accountability & $\begin{array}{l}\text { Transparência } \\
\text { Responsabilização } \\
\text { Prestação de contas }\end{array}$ & $\begin{array}{c}21 \\
9 \\
3\end{array}$ & $\begin{array}{l}95 \% \\
41 \% \\
14 \%\end{array}$ \\
\hline
\end{tabular}

Fonte: dados da pesquisa. 
A partir da análise das entrevistas com especialistas e gestores, um instrumento de avaliação foi construído e submetido à validação de juízes. A Tabela 3 apresenta os coeficientes de cada critério em cada um dos itens submetidos à análise.

Tabela 3 | Coeficientes de validação dos itens

CVCc

\begin{tabular}{|c|c|c|c|c|}
\hline Item & Clareza & $\begin{array}{l}\text { Pertinência } \\
\text { prática }\end{array}$ & $\begin{array}{l}\text { Relevância } \\
\text { teórica }\end{array}$ & $\begin{array}{l}\text { Média dos } \\
\text { critérios }\end{array}$ \\
\hline 1 & 0,74 & 0,91 & 0,94 & 0,87 \\
\hline 2 & 0,77 & 0,91 & 0,91 & 0,87 \\
\hline 3 & 0,71 & 0,89 & 0,89 & 0,83 \\
\hline 4 & 0,77 & 0,91 & 0,91 & 0,87 \\
\hline 5 & 0,97 & 0,97 & 0,97 & 0,97 \\
\hline 6 & 0,51 & 0,83 & 0,90 & 0,75 \\
\hline 7 & 0,57 & 0,77 & 0,77 & 0,70 \\
\hline 8 & 0,77 & 0,89 & 0,89 & 0,85 \\
\hline 9 & 0,80 & 0,89 & 0,94 & 0,88 \\
\hline 10 & 0,80 & 0,89 & 0,94 & 0,88 \\
\hline 11 & 0,94 & 0,94 & 0,97 & 0,95 \\
\hline 12 & 0,86 & 1,00 & 1,00 & 0,95 \\
\hline 13 & 0,74 & 0,94 & 0,97 & 0,89 \\
\hline 14 & 0,83 & 0,94 & 0,94 & 0,90 \\
\hline 15 & 0,80 & 0,94 & 1,00 & 0,91 \\
\hline 16 & 0,86 & 0,91 & 0,94 & 0,90 \\
\hline 17 & 0,74 & 0,91 & 0,91 & 0,86 \\
\hline 18 & 0,91 & 0,94 & 1,00 & 0,95 \\
\hline 19 & 0,89 & 0,94 & 0,97 & 0,93 \\
\hline 20 & 0,83 & 0,94 & 0,97 & 0,91 \\
\hline 21 & 0,80 & 0,94 & 0,97 & 0,90 \\
\hline 22 & 0,80 & 0,89 & 0,91 & 0,87 \\
\hline 23 & 0,69 & 0,77 & 0,91 & 0,79 \\
\hline
\end{tabular}


CVCc

\begin{tabular}{|c|c|c|c|c|}
\hline Item & Clareza & $\begin{array}{l}\text { Pertinência } \\
\text { prática }\end{array}$ & $\begin{array}{l}\text { Relevância } \\
\text { teórica }\end{array}$ & $\begin{array}{l}\text { Média dos } \\
\text { critérios }\end{array}$ \\
\hline 24 & 0,69 & 0,83 & 1,00 & 0,84 \\
\hline 25 & 0,77 & 0,83 & 1,00 & 0,87 \\
\hline 26 & 0,91 & 0,91 & 0,97 & 0,93 \\
\hline 27 & 0,91 & 0,94 & 0,97 & 0,94 \\
\hline 28 & 0,83 & 0,93 & 0,80 & 0,86 \\
\hline 29 & 0,63 & 0,80 & 0,80 & 0,74 \\
\hline 30 & 0,83 & 0,97 & 0,83 & 0,88 \\
\hline 31 & 0,86 & 0,94 & 0,94 & 0,91 \\
\hline 32 & 0,80 & 0,94 & 0,94 & 0,90 \\
\hline 33 & 0,91 & 0,97 & 1,00 & 0,96 \\
\hline 34 & 0,91 & 0,97 & 1,00 & 0,96 \\
\hline 35 & 0,74 & 0,86 & 1,00 & 0,87 \\
\hline 36 & 0,74 & 0,86 & 1,00 & 0,87 \\
\hline 37 & 0,91 & 0,83 & 0,86 & 0,87 \\
\hline 38 & 0,77 & 0,94 & 1,00 & 0,90 \\
\hline 39 & 0,77 & 0,97 & 0,97 & 0,90 \\
\hline 40 & 0,91 & 0,89 & 0,89 & 0,90 \\
\hline 41 & 0,89 & 0,97 & 0,97 & 0,94 \\
\hline 42 & 0,77 & 0,89 & 0,97 & 0,88 \\
\hline 43 & 0,83 & 0,87 & 1,00 & 0,90 \\
\hline 44 & 0,89 & 0,87 & 1,00 & 0,92 \\
\hline 45 & 0,74 & 0,87 & 0,97 & 0,86 \\
\hline 46 & 0,83 & 0,87 & 1,00 & 0,90 \\
\hline 47 & 0,71 & 1,00 & 1,00 & 0,90 \\
\hline 48 & 0,71 & 1,00 & 1,00 & 0,90 \\
\hline 49 & 0,87 & 1,00 & 1,00 & 0,96 \\
\hline 50 & 0,83 & 0,91 & 0,91 & 0,89 \\
\hline
\end{tabular}




\begin{tabular}{lcccc}
\hline Item & Clareza & $\begin{array}{c}\text { Pertinência } \\
\text { prática }\end{array}$ & $\begin{array}{c}\text { Relevância } \\
\text { teórica }\end{array}$ & $\begin{array}{c}\text { Média dos } \\
\text { critérios }\end{array}$ \\
\cline { 2 - 5 } $\mathbf{5 1}$ & 0,74 & 0,94 & 1,00 & $\mathbf{0 , 9 0}$ \\
\hline $\mathbf{5 2}$ & 0,91 & 0,97 & 1,00 & $\mathbf{0 , 9 6}$ \\
\hline $\mathbf{5 3}$ & 0,91 & 0,97 & 0,97 & $\mathbf{0 , 9 5}$ \\
\hline $\mathbf{5 4}$ & 0,94 & 0,97 & 1,00 & $\mathbf{0 , 9 7}$ \\
\hline $\mathbf{5 5}$ & 0,80 & 0,89 & 0,89 & $\mathbf{0 , 8 6}$ \\
\hline $\mathbf{5 6}$ & 0,94 & 0,89 & 0,89 & $\mathbf{0 , 9 0}$ \\
\hline $\mathbf{5 7}$ & 0,83 & 0,89 & 0,89 & $\mathbf{0 , 8 7}$ \\
\hline $\mathbf{5 8}$ & 0,71 & 1,00 & 1,00 & $\mathbf{0 , 9 0}$ \\
\hline CVCt & $\mathbf{0 , 8 1}$ & $\mathbf{0 , 9 1}$ & $\mathbf{0 , 9 5}$ & $\mathbf{0 , 8 9}$ \\
\hline
\end{tabular}

Fonte: dados da pesquisa.

Como se observa na última linha da tabela, o coeficiente total (CVCt) dos critérios quanto à clareza de linguagem $(0,81)$, pertinência prática $(0,91)$ e relevância teórica $(0,95)$ apresentaram valores de validação considerados entre satisfatórios (valor entre 0,80 e 0,90 ) e excelentes (acima de 0,90), podendo-se afirmar a adequação geral do instrumento de avaliação da governança.

Os comentários espontâneos dos juízes foram analisados e subsidiaram a tomada de decisão para ajustes nos itens de instrumentos, seja em relação à alteração textual, situação em que a redação dos itens foi modificada para atribuir maior clareza ao que se espera mensurar; à manutenção, quando o item foi mantido integralmente por apresentar coeficiente de clareza acima de 0,80 e/ou quando não houve comentários dos juízes; à exclusão, itens com média de coeficiente abaixo de 0,80; e inclusão, nos casos em que houve recomendação para divisão dos itens que apresentaram dualidade de resposta.

Após os devidos ajustes a partir da validação do instrumento, apresenta-se a proposta de questionário de avaliação da governança organizacional para a organização pública. A escala utilizada foi do tipo Likert numérica e contínua de sete pontos, de 1 a 7, em que 1 refere-se ao menor nível de concordância (discordo totalmente) e 7 o maior 
nível de concordância (concordo totalmente) sobre o item. Cada item foi elaborado a partir da descrição apresentada nos textos obtidos na revisão de literatura.

\section{Quadro 2 | Instrumento de avaliação da governança organizacional}

Na minha organização...

Existem Comitês Estratégicos ou unidades similares de apoio à governança.

(Por Comitês Estratégicos ou unidades de apoio à governança

1 entenda-se instâncias administrativas, como um Comitê (1) (2) (3) (4) (5) (6) (7) composto por diretores, que dão suporte ao Presidente ou dirigente máximo da organização para a realização dos objetivos institucionais).

2 A Auditoria Interna atua como instância de controle na organização.

(1) (2) (3) (4) (5) (6) (7)

3 A Auditoria Interna atua como instância de governança na organização.

(1) (2) (3) (4) (5) (6) (7)

A gestão da informação é realizada para manter a continuidade das atividades. (Por gestão da informação

4 entenda a padronização e registro das rotinas, dos processos

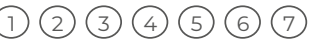
de trabalho, a formalização de manuais e procedimentos, o mapeamento de processos).

A comunicação interna acontece de forma fluida. (Por comunicação interna entenda o compartilhamento de

5 informações institucionais na organização e fluidez, quando há transmissão e possibilidade de retorno sobre a informação).

Os departamentos atuam de forma coordenada para o alcance dos objetivos institucionais.

(1) (2) (3) (4) (5) (6) (7)

A infraestrutura tecnológica permite fazer a gestão da

7 informação. (Por infraestrutura tecnológica entenda a disponibilidade de máquinas, equipamentos, sistemas, banco de dados, rede, serviços).

8 A estratégica de atuação está definida.

9 A estratégia de atuação é executada.

10 Há clareza dos resultados a serem alcançados.

11 As pessoas conhecem a missão institucional. 
Na minha organização...

É assegurada a alocação eficiente dos recursos (financeiros,

12 materiais, humanos e tecnológicos) para desenvolvimento

(1) (2) (3) (4) (5) (6) (7) das atividades.

13 Há objetivos estratégicos definidos.

(1) (2) (3) (4) (5) (6) (7)

14 Há Planejamento Estratégico formalizado.

(1) (2) (3) (4) (5) (6) (7)

15 O planejamento das atividades é revisado periodicamente.

(1) (2) (3) (4) (5) (6) (7)

Realiza-se avaliação de riscos estratégicos e específicos que possam comprometer o alcance dos objetivos da

16 organização. (Por riscos, entenda a possibilidade de

(1) (2) (3) (4) (5) (6) (7) ocorrência de um evento que venha a ter impacto no cumprimento dos objetivos da organização).

Os canais de comunicação com as partes interessadas estão estabelecidos. (Por partes interessadas entendase: sociedade, pesquisadores, secretarias de governo, instituições de ensino, órgãos de controle, dentre outros que influenciam ou possam influenciar as atividades da organização).

Há divulgação dos canais de comunicação para as partes interessadas.

19 Há incentivo à participação das partes interessadas nas atividades da organização.

Há ações que busquem conscientizar a sociedade quanto à sua participação nas atividades da organização.

21 Há ações com foco no atendimento às necessidades das partes interessadas.

Há parcerias com outras instituições, integrantes do governo, para alcançar seus objetivos institucionais.

Há parcerias com outras instituições, de fora do governo, para alcançar seus objetivos institucionais.

24 As parcerias são formalizadas.

25 São claras as atribuiç̧ões de cada parte nas parcerias.

26 O conhecimento e as práticas adquiridas em regime de parceria são incorporados às atividades.

27 Realiza-se controle de desempenho das parcerias. 
Na minha organização...

A alta direção define as políticas da organização.

28 (Por alta direção entenda-se a autoridade máxima e os (1) (2) (3) (4) (5) (6) (7) dirigentes superiores).

A alta direção promove a gestão participativa na tomada de decisão. (Por gestão participativa considere a atitude gerencial da alta direção que busque o máximo de

29 cooperação das pessoas, reconhecendo a capacidade e o potencial diferenciado de cada um e harmonizando os interesses individuais e coletivos, a fim de conseguir a sinergia das equipes de trabalho).

30 Há processo formalizado de seleção de pessoas do corpo técnico.

(1) (2) (3) (4) (5) (6) (7)

Os requisitos para ocupar cargos de liderança na organização estão formalizados no arranjo institucional.

31 (Por arranjo institucional entenda lei de criação da organização, decreto regulamentador da estrutura).

É realizada a capacitação do corpo técnico para desempenho das atividades.

(1) (2) (3) (4) (5) (6) (7)

33 É realizada a capacitação das lideranças para desempenho das atividades.

(1) (2) (3) (4) (5) (6) (7)

34 É realizada avaliação de desempenho das lideranças.

(1) (2) (3) (4) (5) (6) (7)

35 É realizada avaliação de desempenho do corpo técnico.

São promovidas ações de motivação para atuação na organização.

37 Há prática de recompensa pelo desempenho.

38 Há prática de punição pelo desempenho.

As atuais carreiras incentivam a manutenção dos servidores na organização.

40 As pessoas têm consciência que sua atividade impacta nos resultados da organização.

As atribuições e responsabilidades estão claramente definidas.

42 Atua-se de acordo com a estrutura formalizada.

43 Há comprometimento ético da liderança. 
Na minha organização...

44 Há comprometimento ético do corpo técnico.

(1) (2) (3) (4) (5) (6) (7)

45 Adota-se código de ética adaptado ao contexto de atuação da organização.

(1) (2) (3) (4) (5) (6) (7)

46 O código de ética é aplicado a todos da organização, corpo técnico e lideranças.

(1) (2) (3) (4) (5) (6) (7)

47 Ocorre a transparência das informações ao público interno.

(1) (2) (3) (4) (5) (6) (7)

48 Ocorre a transparência das informações ao público externo.

(1) (2) (3) (4) (5) (6) (7)

49 As informações divulgadas são compreensíveis ao público a que se destina.

(1) (2) (3) (4) (5) (6) (7)

50 Ocorre a transparência na tomada de decisão.

51 Ocorre a avaliação de satisfação das ações desenvolvidas pela organização.

52 O desempenho operacional é monitorado.

53 Os indicadores de desempenho estão definidos.

54 O desempenho operacional é avaliado periodicamente. (1) (2) (3) (4) (5) (6) (7)

55 Há prática de controle da gestão orçamentária e financeira. (1) (2) (3) (4) (5) (6) (7)

56 Os conflitos de interesses que possam comprometer os
objetivos da organização são gerenciados.

58 A prestação de contas à sociedade é feita periodicamente. (1) (2) (3) (4) (5) (6) (7) Fonte: dados da pesquisa.

\section{Discussão}

Para a construção da proposta de instrumento de avaliação de governança foram analisadas as informações obtidas na revisão de literatura (Quadro 1) e dos relatos, individualizados e consolidados, das entrevistas realizadas, bem como dos comentários aportados pelos juízes nos formulários submetidos à validação. Nessa perspectiva, as 
variáveis estratégia da organização e exercício de liderança surgiram, respectivamente, como as primeiras impressões tanto nos discursos dos especialistas quanto nos discursos dos gestores da organização de estudo. Há, portanto, alinhamento entre teoria e o discurso praticado pelos entrevistados de que o conjunto de mecanismos de governança apoia a organização no alcance de seus objetivos institucionais, tendo a liderança uma parcela importante nessa atuação (ANAO, 2014; TCU, 2014).

Ainda em relação à importância das variáveis, observa-se alta frequência das variáveis relacionadas a gestão de pessoas, stakeholders, gestão de riscos, parcerias, liderança e transparência, o que possibilita inferir tais variáveis como as principais de governança organizacional, pois segundo Bardin (2011), a quantidade e a ordem em que elementos de informação aparecem nos discursos dos entrevistados permitem identificar a importância de cada registro.

Em contrapartida, a variável remuneração da alta administração, que é apresentada nos referenciais do TCU (2014) e da International Federation of Accountants (IFAC) (2001), bem como o elemento identificado como configuração do conselho de administração, não foram mencionados nas entrevistas, quer pelo grupo de especialistas, quer por gestores da organização de estudo. Trata-se, portanto, de variáveis não observáveis ao tipo de organização estudada - autarquia - e que foram excluídas da proposta final do instrumento de avaliação.

Seguindo a análise, verifica-se o surgimento de duas novas variáveis - apoio tecnológico e práticas de governança - citadas por $27 \%$ e $14 \%$ de especialistas e gestores, respectivamente. Essas duas variáveis surgiram nos discursos dos entrevistados como dois elementos necessários à boa governança e não estão, ao menos explicitamente, apresentadas nos princípios e práticas de governança dos referenciais analisados no tópico 2 - referencial teórico.

Na variável relativa a apoio tecnológico, a perspectiva dos entrevistados é de que o contexto das organizações se apresenta cada vez mais dinâmico, com a adoção de mecanismos de gestão da informação a serem suportados pelo apoio da tecnologia, o que corrobora o estudo de Zorzal e Rodrigues (2015) sobre a convergência dos princípios de disclosure e transparência à luz dos princípios de boas práticas de governança aplicadas 
ao setor público, evidenciando o uso das tecnologias para impulsionar a produção, a organização, a disseminação da informação e a entrega de accountability de maneira eficaz à sociedade, permitindo, assim, que um stakeholder possa se integrar e avaliar as iniciativas realizadas pela organização. Nessa linha e à luz da teoria institucional, pode-se aventar que as mudanças na governança, motivadas pela gestão da informação, podem decorrer do processo mimético, em que se empregam práticas reconhecidas por outras organizações a fim de mitigar o risco de incerteza.

Os comitês de apoio à governança foram reportados como importantes instâncias administrativas por $32 \%$ dos entrevistados, o que também foi encontrado no estudo realizado por Pascoal e Oliveira (2017), segundo o qual praticamente todos os órgãos da administração pública federal brasileira adotam a prática de instituir uma instância de apoio à governança.

$\mathrm{Na}$ variável processos organizacionais, $82 \%$ dos entrevistados demonstraram a preocupação quanto ao gerenciamento das informações da organização e houve associação desse elemento à capacitação dos colaboradores da organização, porque, segundo um dos especialistas entrevistado, a falta de capacitação dos servidores ocasiona erros não intencionais que resultam, ainda assim, em punição aos servidores.

A variável stakeholders figurou no discurso de todos os entrevistados. Para a maioria dos entrevistados, $82 \%$, a organização deve pautar sua estratégia e ações para atendimento aos interesses do máximo de stakeholders, o que corrobora a teoria dos stakeholders quanto à adoção de estratégias diferenciadas para as diversas partes interessadas da organização (FreEmAn; ReED, 1983; FroomAn, 1999; MAINARDES; Alves; RAPOSO, 2012). Por outro lado, a participação dos stakeholders na gestão da organização, como apontado na literatura (ANAO, 2014; BRASIL, 2016; GNAN et al., 2013; IFAC/CIPFA, 2014; LOI, 2016; OCDE, 2015; TCU, 2014), não foi consenso entre os entrevistados. Um elemento condicionante à participação do stakeholder sociedade na organização de estudo, por exemplo, refere-se à necessidade de conscientização para que esta participe ativamente das iniciativas promovidas pela organização.

Ainda no que se refere à gestão de pessoas, observa-se a necessidade de capacitação contínua das pessoas que atuam na organização, e da realização de uma avaliação efetiva 
da liderança. Considerando que a liderança está associada à eficácia gerencial, que influencia o clima organizacional (PIMENTA; AOUAR; OLIVEIRA, 2012) e que um dos gestores da organização de estudo apresentou desconfiança quanto à efetividade da avaliação de liderança realizada pelo setor de gestão de pessoas da organização, a avaliação das lideranças se apresenta como oportunidade de melhoria para a organização de estudo.

Em relação à variável accountability, verificou-se que a variável transparência, relacionada à exposição da atuação das organizações e dos agentes públicos, foi a que obteve maior menção entre os entrevistados, seguida das variáveis responsabilização e prestação de contas, respectivamente. A ocorrência corrobora a estudo de Koppell (2005) de que a transparência é a categoria mais importante da accountability. Por outro lado, as variáveis apuração de irregularidades e realização de auditoria anual, presentes no modelo de governança (Quadro 1) não foram explicitamente mencionadas pelos dois grupos de entrevistados, o que resultou na exclusão de ambas da proposta de instrumento.

Por fim, ainda em relação à transparência, cabe destacar que, no caso da organização de estudo, os gestores demonstraram a preocupação com a manutenção do sigilo dos dados coletados pela organização e divulgados à sociedade, isso porque a proteção da informação individual deve ser resguardada. Na mesma direção quanto à proteção da informação individual, há o pronunciamento do Tribunal de Contas da União, Acórdão 3.022/2015 - Plenário (TCU, 2015), no sentido de se adotar como boa prática a exclusão ou mascaramento, total ou parcial, de atributos como nome, telefone ou outros documentos de identificação que impeçam a identificação das pessoas.

Nesse sentido, considerando os conceitos de isomorfismos trazidos pela Teoria Institucional e a mudança de comportamento da organização de estudo na divulgação de seus dados, com o devido mascaramento ou proteção dos dados individuais, e, ainda, a recente publicação de lei que trata da proteção dos dados pessoais - Lei no 13.709, de 14/08/2018 (BRASIL, 2018), pode-se entender que a institucionalização de uma transparência relativa, como variável de governança, ou seja, a transparência dos dados com proteção de determinadas informações, tem sido motivada pelo isomorfismo normativo. 


\section{Conclusão}

Este trabalho teve como objetivo apresentar a proposta de instrumento de avaliação da governança organizacional para uma organização pública, o que se concretizou a partir de análise documental, realização de entrevistas, desenvolvimento e validação do instrumento. Inicialmente, os princípios e as práticas de governança aplicáveis ao setor público foram descritos por meio da análise de conteúdo da revisão de literatura e dos principais referenciais de governança, conforme o Quadro 1. Em seguida, foram realizadas entrevistas com especialistas, até encontrar o ponto de saturação teórica (FALQUETTO; FARIAS, 2016), e com gestores da instituição de estudo. A Teoria Institucional e a Teoria dos Stakeholders foram utilizadas como suporte teórico para analisar os resultados dessa pesquisa.

Como resultado, foram observadas poucas alterações entre o modelo de avaliação da governança identificado na literatura e o modelo de governança validado. Como exemplo de não receptividade, tem-se os elementos relacionados ao conselho de administração e remuneração da alta administração. Por outro lado, novos elementos surgiram das entrevistas com especialistas e gestores e foram mantidos após a validação do instrumento de avaliação pelos juízes, caso, por exemplo, dos elementos relacionados a instâncias de apoio à governança e apoio tecnológico.

Sobre o processo de incorporação efetiva das novas práticas de governança, observase que esta ocorrerá quando houver a institucionalização das práticas na organização, o que demandará mudanças na instituição. Tais mudanças têm sido observadas a partir do processo normativo, em que há obrigatoriedade na adoção de ajustes de práticas de governança, especialmente em decorrência das ações dos órgãos de controle, como os tribunais de contas.

Por fim, os resultados discutidos ao longo desse trabalho devem levar em consideração algumas limitações. Uma delas refere-se à impossibilidade de generalização a instituições, especialmente aquelas com finalidade lucrativa, uma vez que se trata de um estudo de caso em uma autarquia federal e, por isso, as situações identificadas podem não ser do contexto de outras organizações (YıN, 2001). 
Como recomendação de estudos futuros, recomenda-se a realização de pesquisas empíricas por meio da aplicação do instrumento de avaliação de governança já validado e a replicação do estudo no contexto de outras organizações públicas.

\section{Referências bibliográficas}

Australian National Audit OfFice (ANAO). Public sector governance: strengthening performance through good governance - better practice guide. June 2014. Disponível em: <https://apo.org.au/ node/40252>. Acesso em 10 de jul. 2016.

BARDIN, Laurence. Análise de Conteúdo. São Paulo: Edições 7, 2011.

Bello, Holtajana. Audit Committee's Role in Enhancing Accountability of the Albanian Public Sector. European Journal of Business and Economics, Albania. v. 8, n. 4, p. 14-19, 2013.

BENEDICTO, Samuel Carvalho de et al. Governança corporativa: uma análise da aplicabilidade dos seus conceitos na administração pública. Organizações Rurais \& Agroindustriais, Lavras, v. 15, n. 2, p. 286-300, 2013.

BLANK, JOS L T; VAN HULST, Bart Laurents. Governance and performance: the performance of Dutch hospitals explained by governance characteristics. Journal of Medical Systems, v. 35, n. 5, p. 991999, 2011.

BRASIL, Lei n. 13.303, de 30 de junho de 2016. Dispõe sobre o estatuto jurídico da empresa pública, da sociedade de economia mista e de suas subsidiárias. Diário Oficial da União, 1/07/2016. Disponível em: <http://www.planalto.gov.br/ccivil_03/_ato2015-2018/2016/lei/ I13303.htm>. Acesso em: 20 dez. 2016.

BRASIL, Lei n. 13.709, de 14 de agosto de 2018. Lei Geral de Proteção de Dados Pessoais (LGPD). Diário Oficial da União, 1/07/2016. Disponível em: http://www.planalto.gov.br/ccivil_03/_ ato2015-2018/2018/lei/L13709.htm. Acesso em: 20 de ago. 2018.

BRASIL, Lei n. 9.448, de 14 de março de 1977. Transforma o Instituto Nacional de Estudos e Pesquisas Educacionais - INEP em Autarquia Federal. Diário Oficial da União, Edição extra, de 15/03/2017. Disponível em: <http://www.planalto.gov.br/ccivil_03/leis/l9448.htm>. Acesso em: 20 out. 2016.

CARVAlho, Cristina Amélia; VieIRA, Marcelo Milano Falcão; SILVA, Sueli Maria Goulart. A trajetória conservadora da teoria institucional. Gestão.Org, v. 10, n. especial, p. 469-496, 2012.

CavalCante, Mônica C.N.; De LuCA, Marcia M.M. Controladoria como instrumento de governança no setor público. Revista de Educação e Pesquisa em Contabilidade, v. 7, n. 1, p. 73-90, 2013.

CHAHAL, Hardeep; KUMARI, Archana. Examining talent management using CG as proxy measure: a case study of State Bank of India. Corporate Governance, v. 13, n. 2, p. 198-207, 2013. 
CGU/MPOG, Controladoria - Geral da União (CGU) e Ministério do Planejamento, Orçamento e Gestão (MPOG). Instrução Normativa n.01. 2016. Disponível em: http://www.cgu.gov.br/sobre/ legislacao/arquivos/instrucoes-normativas/in_cgu_mpog_01_2016.pdf/view. Acesso em 01 de ago. 2016.

Cherman, Andréa; Tomel, Patrícia Amélia. Códigos de ética corporativa e a tomada de decisão ética: instrumentos de gestão e orientação de valores organizacionais? Revista de Administração Contemporânea, v. 9, n. 3, p. 99-120, 2005.

Dimaggio, Paul J.; PowelL, Walter W. The Iron Cage: Institutional Isomorphism and Collective Rationality in Organizational Fields. Amercican Sociological Review, v. 48, n. 2, p. 147-160, 1983.

ElLWOOD, Sheila; GARCIA-LACALLE, Javier. Examining Audit Committees in the Corporate Governance of Public Bodies. Public Management Review, v. 188, n. october, p. 1138-1162, 2015.

FALQUETTO, Júnia; FARIAS, Josivania. Saturação teórica em pesquisas qualitativas: relato de uma experiência de aplicação em estudo na área de administração. CIAQ2016, v.3, p. 560-569. 2016.

Flórez-PARRA, Jesús Mauricio; López-PÉReZ, María Victoria; LóPEZ-HernándeZ, Antonio Manuel. Gobierno corporativo y sector público: un estudio bibliométrico en las principales revistas Isi. Innovar, v. 24, n. 51, p. 79-98, 2014.

FONTES-FILHO, Joaquim Rubens. Governança organizacional aplicada ao setor público. VIII Congreso Internacional del CLAD sobre la Reforma del Estado y de la Administración PúblicaPanamá, n. 2003, p. 28-31, 2003.

FREemAN, R. Edward; ReED, David L. Stockholders and stakeholders: a new perspective on corporate governance. California Management Review, v. 25, n. 3, p. 88-106, 1983.

Frooman, Jeff. Stakeholder Influence Strategies. Academy of Management Review, v. 24, n. 2, p. 191-205, 1999.

GNAN, Luca et al. Corporate governance and management practices: stakeholder involvement, quality and sustainability tools adoption: evidences in local public utilities. Journal of Management and Governance, v. 17, n. 4, p. 907-937, 2013.

GOMES, Ricardo Corrêa. Uma proposta de instrumento de pesquisa para explorar as influências dos stakeholders nas organizações públicas. Alcance, v. 12, n. 1, p. 9-26, 2005.

GRINDLE, Merilee S. Good governance, R.I.P.: a critique and an alternative. Governance: an International Journal of Policy, Administration, and Institutions, v. 30, n. 1, p. 17-22, 2017.

Hernández Nieto, Rafael. Contribuciones al Análisis Estadístico. Mérida, Venezuela: Universidad de Los Andes/IESINFO, 2002.

HoRA, Rejane Angélica de Andrade; OliveIRA, Oderlene Vieira De; FoRTE, Sérgio Henrique Arruda Cavalcante. Governança corporativa no setor público e privado no contexto brasileiro: a produção e a evolução acadêmica em dez anos de contribuições. p. 16. 2014. 
IFAC/CIPFA. International framework: good governance in the public sector, 2014. Disponível em: https://www.ifac.org/publications-resources/international-framework-good-governance-publicsector. Acesso em 10 de jul. 2016.

IFAC, International Public Sector Study. Governance in the Public Sector, 2001. Disponível em: https://www.ipsasb.org/publications-resources/study-13-governance-public-sector. Acesso em 10 de jul. 2016.

JARBouI, Sami; Forget, Pascal; Boujelbene, Younes. Inefficiency of public road transport and internal corporate governance mechanisms. Case Studies on Transport Policy, v. 2, n. 3, p. 153-167, 2014.

KIM, Soojin; KIM, Jeong-Nam. Bridge or buffer: two ideas of effective corporate governance and public engagement. Journal of Public Affairs, v. 16, n. 2, p. 118-127, 2016.

KOPPELL, Jonathan G. S. Pathologies of accountability: ICANN and the challenge of "Multiple Accountabilities Disorder". Public Administration Review, v. 65, n. 1, p. 94-108, 2005.

LoI, Teck Hui. Stakeholder management: a case of its related capability and performance. Management Decision, NULL, v. 54, n. 1, p. 148-173, 2016.

MAINARDES, Emerson. W.; AlVES, Helena; RAPOSO, Mario. A model for stakeholder classification and stakeholder relationships. Management Decision, v. 50, n. 10, p. 1861-1879, 2012.

MARQUES, Maria da Conceição da Costa. Aplicação dos princípios da governança corporativa ao sector público. Revista de Administração Contemporânea, v. 11, n. 2, p. 11-26, 2007.

MARTINS, Humberto Falcão; MARINI, Caio. Governança Pública Contemporânea : uma tentativa de dissecação conceitual. Revista do Tribunal de Contas da União, p. 42-54, 2014.

MAZzıonI, Sady et al. Governança Corporativa : análise bibliométrica do período de 2000 a 2012. REUNIR - Revista de Administração, Contabilidade e Sustentabilidade, v. 5, n. 1, p. 1-20, 2015.

MEINTJES, C.; GRobleR, A. F. Do public relations professionals understand corporate governance issues well enough to advise companies on stakeholder relationship management? Public Relations Review, v. 40, n. 2, p. 161-170, 2014.

Ministério do Planejamento, OrÇamento e Gestão. Programa Gespública, Modelo de excelência em gestão pública. 2014. Disponível em: http://www.gespublica.gov.br/sites/default/files/ documentos/modelodeexcelenciaemgestaopublica2014.pdf. Acesso em 01 de out. 2016.

MitCHeLL, Ronald K; AgLE, Bra; Wood, Donna J. Toward a Theory of Stakeholder identification and salience : defining the principle of who and what really counts authors: Ronald $\mathrm{K}$. Mitchell, Bradley R. Agle and Donna J. Wood Source : the Academy of Management Review , v. 22, n. 4, p. 853-886, 1997.

MSWAKA, Walter; OLU, Aluko. Corporate governance practices and outcomes in social enterprises in the UK. International Journal of Public Sector Management, v. 28, n. 1, p. 57-71, 2015.

NGoepe, Mpho; Ngulube, Patrick. An exploration of the role of records management in corporate governance in South Africa. South African Journal of Information Management, v. 15, n. 2 , p. 1-8, 2013. 
Nkundabanyanga, Stephen Korutaro; Tauringana, Venancio; Muhwezl, Moses. Governing boards and perceived performance of secondary schools. International Journal of Public Sector Management, v. 28, n. 3, p. 221-239, 2015.

OBEROI, Roopinder. Deciphering the diverse nature of gorporate governance in the Indian public sector: a Study of Public Sector Bharat Heavy Electricals Limited (BHEL) Company in David Crowther. The Governance of Risk (Developments in Corporate Governance. v. 5. 2013.

Organisation for ECONOMIC Co-Operation ANd DeVElopment (OECD). Guidelines on Corporate Governance of State-owned Enterprises. 2015. Disponível em: https://www.oecd.org/corporate/ guidelines-corporate-governance-soes.htm. Acesso em 01 de ago. 2016.

OliveIRA, Antônio G.; PISA, Beatriz. J. IGovP : índice de avaliação da governança públicainstrumento de planejamento do Estado e de controle social pelo cidadão. Revista de Administração Pública, v. 49, n. 5, p. 1263-1290, 2015.

PASCOAL, Márcia Néa O.; Oliveira, Oderlene Vieira de. Adoção de melhores práticas de governança na administração pública federal brasileira. Enanpad 2017. p. 0-16. 2017.

PImentA, Raniery Cristiano de Q.; AouAR, Walid Abbas El; OliveIRA, José Arimatés de. Clima organizacional em instituto de pesquisa tecnológica. RaUnp, v. 2, n. IV, p. 23-38, 2012.

PINHO, José Antonio G.; SACRAMENTO, Ana Rita S.. Accountability: já podemos traduzi-la para o português? Revista de Administração Pública, v. 43, n. 6, p. 1343-1368, dez. 2009.

RIBEIRO, Henrique César M. Corporate governance versus corporate governance: an international review: uma análise comparativa da produção acadêmica do tema governança corporativa. Revista Contemporânea de Contabilidade - RCC, v. 11, n. 23, p. 95-116, 2014.

Ribeiro, Henrique César Melo; Machado JunioR, Celso; Souza, Maria Tereza Saraova de; CAMPANÁRIO, Milton de Abreu; CORRÊA, Rosany. Governança corporativa: um Estudo bibliométrico da produção científica das dissertações e teses brasileiras. Contabilidade, Gestão e Governança, v. 15, n. 3, p. 52-70, 2012.

Ribeiro, Henrique César Melo; SAntos, Marianne Corrêa dos. Perfil e evolução da produção científica do tema governança corporativa nos periódicos Qualis / Capes Nacionais: uma análise bibliométrica e de redes sociais. Contabilidade, Gestão e Governança, v. 18, n. 3, p. 4-27, 2015.

RochA, Arlindo, C. Accountability na administração pública: a atuação dos tribunais de contas. Enanpad, 2009. Disponível em: http://www.anpad.org.br/admin/pdf/APS716.pdf. Acesso em 10 de out. 2016.

RoMoliNI, A; GoRI, E; FISSI, S. Public corporate governance and performance information in local utilities: the different perceptions of politicians and managers. Administratie si Management Public, v. 2015, n. 25, p. 55-77, 2015.

SCHELKER, Mark. Auditors and corporate governance: evidence from the public sector. KYKLOS, v. 66, n. 2, p. 275-300, 2013.

SCHIEHLL, Eduardo; MARTINS, Henrique Castro. Cross-national governance research: a systematic review and assessment. Corporate Governance: an International Review, v. 24, n. 3, p. 181-199, 2016. 
SouzA, André Carlos de. Gerenciamento de impressão no Brasil: uma análise das mensagens dos Presidentes publicadas nos relatórios anuais. 2013. Disponível em https://repositorio.ufsc.br/ xmlui/handle/123456789/101070. Acesso em 01 de abr. 2017.

SPANHOVE, Jürgen; VERHOEST, Koen. Corporate governance vs. government governance: translation or adaptation? p.1-40. 2007. Disponível em https://core.ac.uk/download/ pdf/34330232.pdf. Acesso em 01 de nov. 2017

SUBRAMANIAM, Nava et al. Understanding corporate governance in the Australian public sector: a social capital approach. Accounting, Auditing \& Accountability Journal, v. 26, n. 6, p. 946-977, 2013.

TRIBUnal de Contas da União (TCU). Referencial Básico de Governança. Tribunal de Contas da União. 2014. Disponível em: https://portal.tcu.gov.br/governanca/entendendo-a-governanca/ referencial-de-governanca/. Acesso em 10 de jul. 2016.

TRIBUnAL de ContAS dA UNIÃo (TCU). Acórdão 3.022, de 25 de novembro de 2015,Plenário. Disponívelem: https://contas.tcu.gov.br/etcu/AcompanharProcesso?p1=8801\&p2=2015\&p3=0. Acesso em 10 de jul 2016.

ThIRY-Cherques, Hermano Roberto. Saturação em pesquisa qualitativa: estimativa empírica de dimensionamento. Revista PMKT, v. 3, n. outubro, p. 20-27, 2009.

YIN, Robert K. Estudo de caso: planejamento e métodos. Porto Alegre: v. 1. 2001.

ZORZAL, Luzia; RODRIGUES, Georgete Medleg. Disclosure e transparência no setor público: uma análise da convergência dos princípios de governança. Informação \& Informação, v. 20, n. 3, p. 113, 2015.

\section{Nildete dos Passos Oliveira}

(iD) https://orcid.org/0000-0003-0459-0696

Mestre em Administração Pública (UnB, 2017), especialista em Educação a Distância (UnB, 2008). Bacharel em Relações Internacionais (Unieuro, 2006). Pesquisadora-Tecnologista em Informações e Avaliações Educacionais no Instituto Nacional de Estudos e Pesquisas Educacionais Anísio Teixeira (Inep), em exercício na unidade de Auditoria Interna.

E-mail: nildete.oliveira@gmail.com

\section{Pedro Carlos Resende Junior}

(D) https://orcid.org/0000-0002-4220-8243

Doutor e Mestre em Administração pela Universidade de Brasília (UnB). Pós-Doutor em Gestão da Inovação pela Universidade do Minho. Professor do Mestrado Profissional em Administração (PPGA/ UnB). Pesquisador Colaborador Pleno da Universidade de Brasília (UnB). Especialista em Engenharia da Informação. Especialista em Qualidade e Produtividade. Especialista em Marketing. Bacharel em Tecnologia de Processamento de Dados.

E-mail: pcrj73@gmail.com 